\title{
Plasmonic and Hydrodynamic Effects in Ultrafast Laser-Induced Periodic Surface Structures on Metals
}

\author{
J.P. Colombier*1 ${ }^{1}$, F. Garrelie $*^{1}$, P. Brunet $*^{2}$, A. Bruyère* ${ }^{1}$, F. Pigeon* ${ }^{1}$, R. Stoian* ${ }^{1}$, O. Parriaux $*^{1}$ \\ ${ }^{* 1}$ Laboratoire Hubert Curien (UMR 5516 CNRS), Université de Lyon, Université de Saint-Etienne, \\ 42000 Saint Étienne, France \\ E-mail: Jean.philippe.colombier@univ-st-etienne.fr \\ ${ }^{* 2}$ CNRS, Laboratoire Matière et Systèmes Complexes, UMR 7057 CNRS \& Université Paris 7 , \\ UMR 7057, F-75205 Paris 13, France
}

\begin{abstract}
We report results on the development of laser-induced periodic surface structures produced by ultrashort laser pulses irradiating metallic surfaces. The surface topology features are discussed in terms of periodicity and amplitude contrast of the pattern formation, and in relation to the chronological sequence of laser-induced events. Resonant excitation of Surface Plasmons in metallic gratings show that the surface wave excited during the femtosecond laser pulse can initiate the observed patterning. Metallic behavior under nonequilibrium conditions on the picosecond timescale is then investigated to correlate the amount of material experiencing solid-to-liquid transitions and the subsequent structure amplitude. With the derived observation, the calculation of the transient nonequilibrium thermodynamic characteristics of excited nickel is performed, allowing to define characteristic timescales of thermocapillary processes which may occur under multi-pulse irradiation.

DOI; 10.2961/jlmn.2012.03.0023
\end{abstract}

Keywords: Ultrafast LIPSS formation ; ripples on metal surface ; femtosecond laser irradiation ; surface plasmon excitation ; thermocapillary effect ; Marangoni effect ; Sipe model ; efficacy factor

\section{Introduction}

The application potential for ultrashort laser pulses relies on their remarkable capacity to confine and localize energy on the smallest scales. In addition, strong energy localization can trigger nanoscale self-arrangement of matter on irradiated surfaces [1-7]. Appearing for fluences around the phase transformation threshold and developing with varying periodicity and amplitudes, the manifestation of periodic surface patterns with subwavelength dimensions, usually known as Laser-Induced Periodic Surface Structures (LIPSS), is presently a less understood but inherent phenomenon. Surpassing the diffraction limit in particular conditions, ultrafast laser excitation can structure well below the incident wavelength. Two distinct types of LIPSS are typically observed when solid materials are irradiated upon ultrashort multiple pulses: Low Spatial Frequency LIPSS (LSFL) and High Spatial Frequency LIPSS (HSFL) [8-12]. The microscopic mechanism of LIPSS formation is still under discussion and various kinds of surface wave excitation methods have been proposed so far besides the conventional interference pattern of the incident or refracted light and scattered wave [13-17]. Whereas ultrafast laser excitation stage is the most investigated mechanism in recent literature, an emphasis has to be put on the post-exposure surface dynamics in this particular irradiation regime to explain the subsequent spontaneous formation of nanostructured patterns.

Therefore, we describe here several approaches to shine light on different aspects of interaction. A comprehension effort related to laser excitation mechanisms is presented, able to assist and validate experiments performed on metals. In this context, we investigate hypotheses assuming that LSFL, having a spatial period close to the irradiation wavelength, are formed due to optical interference of the incident laser radiation with a Surface Plasmon (SP). HSFL formation mechanism is still quite controversial for reasons related to its polarization dependence and it will not be discussed in this paper. If the most part of experiments are performed on a practically smooth surface, we will also present irradiation results obtained on pre-structured surfaces. Grating-coupled surface plasmon resonance on metallic surface has been exploited to investigate the correlation between LIPSS formation under ultrashort laser exposure and SP generation conditions. For Transverse Magnetic (TM) irradiation conditions, a well-defined period of grating was a seed for low spatial frequency LIPSS formation [18]. To design matter transformation patterns with predictable properties for a wide range of materials, the amplitude of the nanostructuring process is another key parameter [5,19-20]. Following localized photoexcitation, the sub-surface region undergoes transient melting and ultra-rapid quenching at the nanoscale. A thorough understanding of the involved physical mechanisms during the matter transformation will therefore help to control LIPSS morphology. Measuring the amplitude contrast of LIPSS obtained on nickel and copper surfaces which are supposed to have different behavior under nonequilibrium conditions, the effects of energy coupling and relaxation strength have been correlated to LIPSS formation [20]. The amount of material experiencing solid-to-liquid transitions appears to 
be correlated to the LIPSS amplitude. Finally, the characteristic development time of LIPSS formation will be discussed in the context of matter transformation phases using a hydrodynamic approach. Simulations of laser-matter interaction, coupling hydrodynamics and heat transfer, will be presented to estimate laser energy deposition, electronion nonequilibrium stage, material heating and growth. The subsequent phase transformation and the lifetime of the molten region can have implications on thermocapillary phenomena. This will be effectively estimated by applying characteristic timescales framing the positive feedback of ripple growth under multipulse irradiation. Finally, the depth of the formed pattern will also affect the grating coupling varying LIPSS periodicity via electromagnetic feedback effects.

\section{Plasmonic response defining LIPSS periodicity}

As LIPSS performed on absorbing materials such as metals or excited semiconductors have been mostly found to grow along the direction perpendicular to the laser polarization, LSFL has been early attributed to the excitation of surface plasmon polaritons [8,21]. A photon cannot transform into a SP without some form of momentum conservation. Since a rough surface corresponds to a superposition of a number of different gratings, the mechanism underlying the coupling of SP on a rough interface is essentially the same as that via a single corrugation [22]. It is well known that in the specific case of a resonance harmonic grating, the incident laser wave impinging on the grating at a certain angle $\theta$ transforms into a diffracted wave propagating along the grating surface as an SP wave. The corresponding phasematching condition then reads as a function of the diffraction order $n, \boldsymbol{k}_{\mathrm{SP}}=\boldsymbol{k}_{\boldsymbol{t}} \pm n \boldsymbol{q}_{\boldsymbol{g}}$, where $\boldsymbol{k}_{\boldsymbol{t}}$ is the surface projection of the laser wave vector $\left|\boldsymbol{k}_{\boldsymbol{t}}\right|=\left|\boldsymbol{k}_{0}\right| \sin \theta=0$ at normal incidence and $\boldsymbol{q}_{g}$ is the main wave vector of the surface roughness, corresponding to the wave vector of the prestructured grating in the experiment described below. The proposed method allows correlating the excitation of plasmons in the optical domain to the formation of LIPSS. We have investigated the formation of LIPSS on pre-structured nickel surfaces having periodic gratings with a prechosen range of periodicities upon irradiation by single linearly polarized Ti:sapphire laser pulses $(800 \mathrm{~nm}, 150 \mathrm{fs})$ in air as a function of the grating period and orientation of the polarization [18]. Considering only the first diffraction order, $n$ $=1$, the condition of phase-matching derived previously simplifies to $\boldsymbol{k}_{\mathrm{SP}}=\boldsymbol{q}_{g}$, describing the diffractive excitation of an SP wave with a wavelength equal to the grating spacing. For the experimental part, a Mach-Zehnder interference scheme has been used to create a number of corrugation gratings on nickel plates coated with a thin photoresist deposited on a Ni substrate by spin coating. Different grating periods $\Lambda_{\mathrm{g}}$ ranging from $440 \mathrm{~nm}$ to $800 \mathrm{~nm}$, with $10 \mathrm{~nm}$ increment, have been fabricated. Each structured surface was then irradiated by single fs laser pulse. Fig. 1a) shows an example of periodic nanorelief written on a Ni sample before irradiation. A maximal coupling is expected for a grating wavelength given by [23]:

$$
\Lambda_{g}^{m}=\frac{2 \pi}{\left|q_{g}^{m}\right|}=\Lambda_{S P}=\lambda \cdot\left\{\mathfrak{R}\left[\sqrt{\frac{\tilde{n}^{2}}{1+\tilde{n}^{2}}}\right]\right\}^{-1},
$$

where $\tilde{n}$ is the optical index of the material. From the complex optical index of Ni, $\tilde{n}=2.48+i 4.38$ given in Ref. [24], we estimate that the maximal coupling should occur at $\Lambda_{g}^{m} \approx 790 \mathrm{~nm}$. To show that the grating of $10 \mathrm{~nm}$ thickness does not modify the expected resonant period, we have calculated the $0^{\text {th }}$-order reflection efficiency. This calculation is performed for an incident TM wave at 800 $\mathrm{nm}$ irradiating a Ni surface grating of $10 \mathrm{~nm}$ depth by Rigorous Coupled Wave Analysis (RCWA) [25]. A rectangular profile of the grooves has been assumed, according to Atomic Force Microscopy (AFM) measurements of the gratings. Fig. 1b) illustrates the $0^{\text {th }}$-order reflection efficiency response; it can be seen that the maximal resonant coupling is close to the expected value of $790 \mathrm{~nm}$, for this relatively low grating depth. Consequently, it can be assumed that the resonant position is not affected by this relatively small grating depth as will be discussed in more details in section 4 .
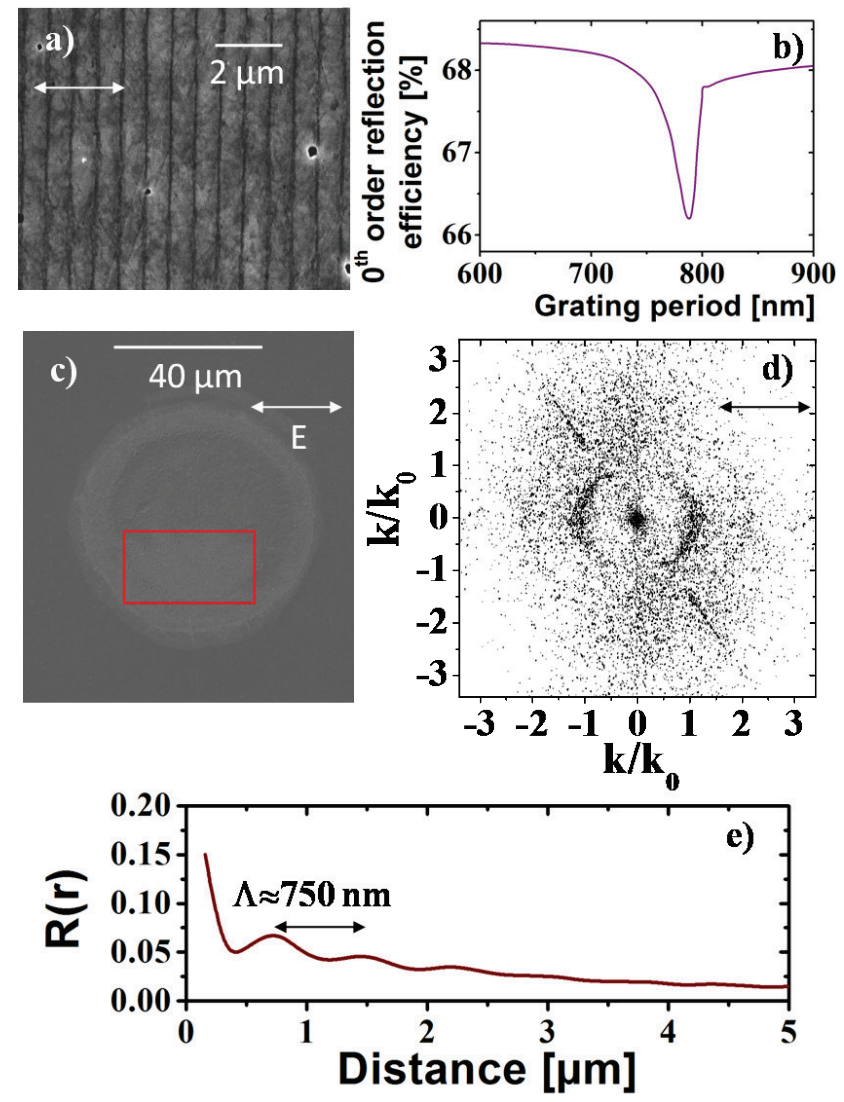

Fig. 1: Grating-coupled surface plasmon enhanced excitation. Example of a representative pre-structured surface at $750 \mathrm{~nm}$ (a). $0^{\text {th }}$ order reflection efficiency calculated by RCWA showing a resonance expected for $\Lambda_{g}^{m} \approx 790 \mathrm{~nm}$ (b). SEM image of the LIPSS formation observed for the specific grating period $\Lambda_{g}^{m} \approx 750 \mathrm{~nm}$ (c) and corresponding 2D-FT image (d). Radially averaged autocorrelation function $R(r)$ processed from the selection on the SEM image (e). 
The irradiation of each grating by a single pulse at a fluence of $1.42 \mathrm{~J} / \mathrm{cm}^{2}$ for the TM and the Transverse Electric (TE) polarizations and at normal incidence reveals that LIPSS formation only occurs for TM irradiation (i.e. the polarization direction perpendicular to the grating structures) and a well defined grating period of $\Lambda_{g}^{m} \approx 750 \mathrm{~nm}$.

Fig. 1c) shows the LIPSS structure generated for this grating periodicity. Single pulse irradiation allows achieving only the early stage of ripple formation and we observe that the pattern is not homogeneously distributed in the irradiated spot. 2D Fourier transform (2D-FT) on the selected part of the acquired image is displayed in Fig. 1d), showing a vertical sickle-shaped feature confirming that LIPSS are perpendicular to the laser polarization as expected for LSFL on metals. The Scanning Electron Microscopy (SEM) image and the corresponding 2D-FT reveal that the period of the formed LIPSS is $\Lambda \approx 750 \mathrm{~nm}$. This result indicates that the specific grating period for which the SP mode is efficiently excited is correlated to the LIPSS formation. The observed resonance is however different from the expected one around $790 \mathrm{~nm}$. Hence, it is supposed that the ultrafast material excitation affects the dielectric properties during the laser pulse, modifies the plasmonic dispersion relation and thus results in a different grating periodicity for which resonance occurs.
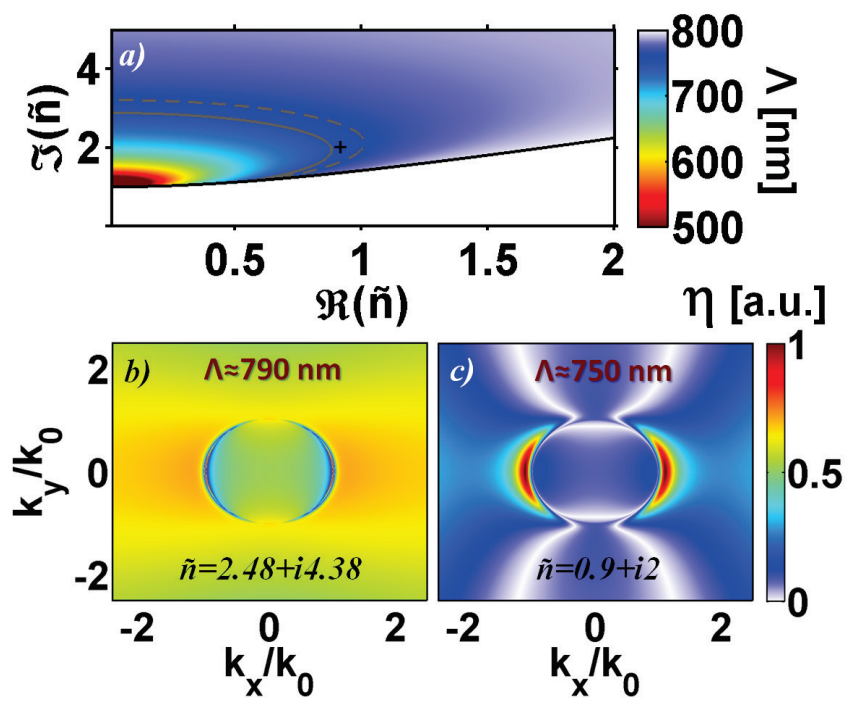

Fig. 2 : Surface plasmon wavelength contour plot as a function of real part and imaginary part of $\tilde{n}$ (a). Grey contour lines marks $\Lambda_{\mathrm{SP}}=750 \mathrm{~nm}$ (solid) and $\Lambda_{\mathrm{SP}}=760 \mathrm{~nm}$ (dashed). Black point indicates the optical index used for excited material. Calculated efficacy factor $\eta$ as a function of the normalized wave vector components $k_{x} / k_{0}$ and $k_{y} / k_{0}$ for unexcited (b) and excited nickel (c).

A radially averaged autocorrelation function $R(r)$ has also been calculated in the direct-space to take into account the $\boldsymbol{k}$ vectors concentrated along the arcs in a $\left(\boldsymbol{k}_{\boldsymbol{x}}, \boldsymbol{k}_{\boldsymbol{y}}\right)$ plane. $R(r)$ is calculated from the autocorrelation of pixels of the binary SEM image as a function of the radial distance $r$ [26]. The calculated function is displayed on Fig. 1e). The oscillating behavior of $R(r)$ is characteristic of a long-range order with periodicity $\Lambda \approx 750 \mathrm{~nm}$, which is more difficult to observe in the SEM image in Fig. 1(c). The decay of the oscillation amplitude is mainly due to features such as ir- regular orientation, bifurcation and limited periodic structures. However, the correlation of the LIPSS appears to extend over several periods. The observed spatial periods of the LIPSS, somewhat smaller than the expected one, can be explained by transient changes of the dielectric properties of the laser-excited material $[8,18,22]$. To illustrate this, we have calculated SP wavelength for a range of optical indices. Fig. 2a) shows a mapping of the solution of eq. (1) as a function of real and imaginary part of $\tilde{n}$. Grey lines point out the required optical index allowing to reach $\Lambda_{\mathrm{SP}}=\Lambda_{\mathrm{g}} \approx 750 \mathrm{~nm}$. White region at the bottom part of the figure indicates the region where surface plasmon cannot be excited, since the condition $\mathfrak{R}\left(\widetilde{n}^{2}\right)<-1$ is not fulfilled [23].

On a non pre-structured metallic surface, SP can be efficiently launched by the natural surface roughness. The propagation of SP and the incident laser wave can interfere to form a periodic intensity distribution. Sipe et al. has proposed a model to describe the inhomogeneous energy absorption resulting from this phenomenon as a function of the laser incidence angle $\theta$, polarization and material dielectric properties [13]. A surface-scattered wave was presumed to be generated at the surface, as observed in our experiment. In the proposed calculation, the grating vector components of the LIPSS are mainly determined by the efficacy factor $\eta\left(\boldsymbol{k} / \boldsymbol{k}_{\boldsymbol{0}}\right)$, depending on a wave vector parallel to the surface $(\boldsymbol{k})$ normalized to the incident laser wave vector $\boldsymbol{k}_{\boldsymbol{0}}$. This function exhibits some sharp peaks which can be associated to dominating LIPSS wave vector and providing an estimation of their spatial period in addition to their main orientation. We have calculated the efficacy factors as a function of the normalized vectors in the surface plane with selvedge parameters identical to the ones given in Ref. [13]. The results are shown in Fig. 2b) for an optical index corresponding to an unexcited material $\tilde{n}=2.48+i 4.38$ and in Fig. 2c) for an excited one with $\tilde{n}=0.9+i 2$. Note that several pairs of real and imaginary parts of $\tilde{n}$ are able to reproduce a SP period $\Lambda \approx 750 \mathrm{~nm}$ as indicated on Fig. 2a) and we have chosen one consistent with a laser-induced electronic excitation. To this, a DrudeLorentz model was applied with various relative intraband and interband contributions, showing that a decrease of the interband transition part could reduce SP wavelength [18]. A normalization of $\eta$ has been performed for each case. Both efficacy factor calculations clearly show the LIPSS perpendicular to the laser electric field since $\eta\left(\boldsymbol{k} / \boldsymbol{k}_{\boldsymbol{0}}\right)$ contains small kinks on Fig. 2b) along $x$ direction superimposed on a varying background and sharp peaks on Fig. 2c). Both forms a sickle-like dominating structure for $\left(k_{x}^{2}+k_{y}^{2}\right)^{1 / 2} \approx 2 \pi / \Lambda_{S P}$ as observed in the Fourier transform shown in Fig. 1d). The resonant LIPSS are related to the excitation of surface plasmons and the observed periods are the same as those discussed previously: $\Lambda \approx 790 \mathrm{~nm}$ in the case of unexcited $\mathrm{Ni}$ and a well pronounced peak at $\Lambda \approx 750 \mathrm{~nm}$ in the case of excited $\mathrm{Ni}$. 


\section{Relaxation of the energy during nonequilibrium and hydrodynamic stages}

The plasmonic approach presented in the first section establishes preliminary steps defining orientation and energy modulation guiding the mechanisms of LIPSS formation. Once the process is triggered, further steps towards nanostructuration follow and several processes are responsible for the final morphology. They require extended approach defining the role of material response from a thermodynamic and hydrodynamic perspective. In particular, material behaviour is strongly dependent on the electronic response upon ultrafast excitation. The effect of the laser irradiation with the response of the material is commonly described by the two-temperature model (TTM). The energy balance between the electron cloud and lattice is described by the temporal evolution of the electron and the lattice temperatures through a coupling parameter $\gamma$ related to the electron-phonon scattering rate and by thermal diffusion processes occurring within each subsystems. The presence of a transient nonequilibrium stage has strong implications on the energy distribution and thus on the final morphology. As energy relaxation dynamics are material dependent, we generated LIPSS on representative materials with different electronic configuration in order to investigate the influence of $\gamma$ and thermal diffusion efficiency on the specific amplitude of LIPSS. Metals with a large range of coupling strengths were exposed to ultrafast laser excitation and the patterned surfaces were investigated ex-situ by AFM and SEM [20].
$\mathrm{Ni}$
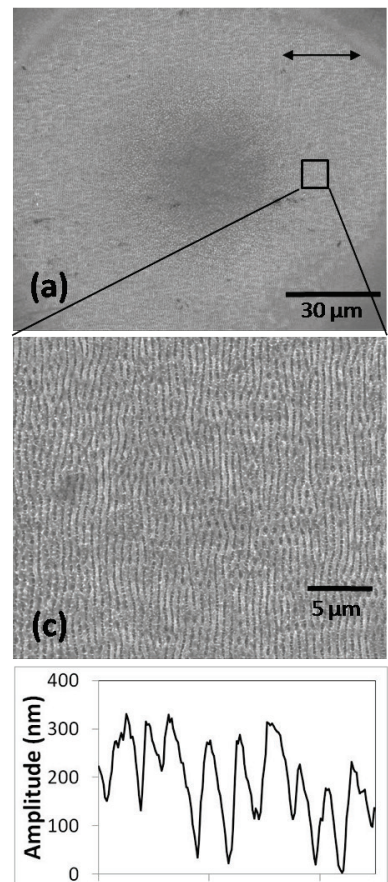

(e)
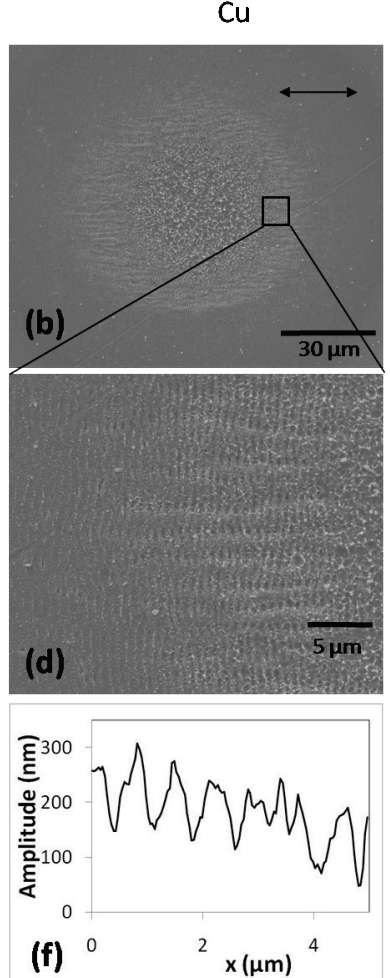

Fig. 3 : SEM images of the LIPSS performed by $\mathrm{N}=50$ laser pulses $(150 \mathrm{fs})$ at a pulse energy of $80 \mu \mathrm{J}$, on $\mathrm{Ni}(\mathrm{a}, \mathrm{c})$ and $\mathrm{Cu}(\mathrm{b}, \mathrm{d})$ and analysis of LIPSS morphology on the different materials: profile lines from AFM images, showing the cross-section of the LIPSS topography for $\mathrm{Ni}(\mathrm{e})$ and $\mathrm{Cu}$ (f). The electric field direction is indicated by the arrow.
The experimental results given in Fig. 3 were obtained for $\mathrm{Ni}$ and $\mathrm{Cu}$ irradiated by 50 pulses of $80 \mu \mathrm{J}(150 \mathrm{fs})$ at $800 \mathrm{~nm}$. The apparent spot size obtained in the case of nickel appears much wider than for copper due to different absorption coefficients. Nevertheless, the absorbed fluence allows to generate LIPSS at the periphery of the focal spot. Fig. 3 (a-d) shows SEM image of the nanostructured region. The relative strength and the electron temperature $\left(T_{e}\right)$ dependence of the electron-phonon coupling in the metals were identified as key factors affecting the initial energy redistribution and the dimensional size of the region undergoing transient melting and resolidification. It appears that LIPSS growth is correlated to the value of the coupling constant and to the amount of material experiencing solidto-liquid transitions.

The LIPSS amplitude measured by AFM is given in Fig. 3 (e) for $\mathrm{Ni}$ and Fig. 3 (f) for $\mathrm{Cu}$. Measurements have been performed at a similar local fluence, close to $0.4 \mathrm{~J} . \mathrm{cm}^{-2}$. The amplitude varies as a function of the material since $\mathrm{Ni}$ has an averaged peak-to-peak amplitude $H \approx 200 \mathrm{~nm}$ while $\mathrm{Cu}$ presents a nearly two fold lower height $H \approx 100 \mathrm{~nm}$. These two materials differ mainly by their electron-phonon coupling constant $\gamma$ and also their thermal conductivity $K$. At room temperature, $\gamma_{\mathrm{Cu}}=5 \times 10^{16} \mathrm{~W} \cdot \mathrm{m}^{-3} \cdot \mathrm{K}^{-1}, K_{\mathrm{Cu}}=398 \mathrm{~W} \cdot \mathrm{m}^{-}$ ${ }^{1} \cdot \mathrm{K}^{-1}$ and $\gamma_{\mathrm{Ni}}=1 \times 10^{19} \mathrm{~W} \cdot \mathrm{m}^{-3} \cdot \mathrm{K}^{-1}, K_{\mathrm{Ni}}=91 \mathrm{~W} \cdot \mathrm{m}^{-1} \cdot \mathrm{K}^{-1}$. Based on electronic energy distribution related to the TTM scenario, a high value of $\gamma$ and a low value of $K$ cause a strong energy confinement. From the strong different values of $\gamma$ and $K$ of $\mathrm{Ni}$ and $\mathrm{Cu}$ we can conclude that the energy confinement after excitation strongly determines the surface modulation amplitude. Therefore, this confinement results in strong longitudinal temperature gradients in a thin molten layer which, in turn, via a corresponding pressure distribution modulates perpendicular surface expansion and thus, finally, results in LIPSS formation.

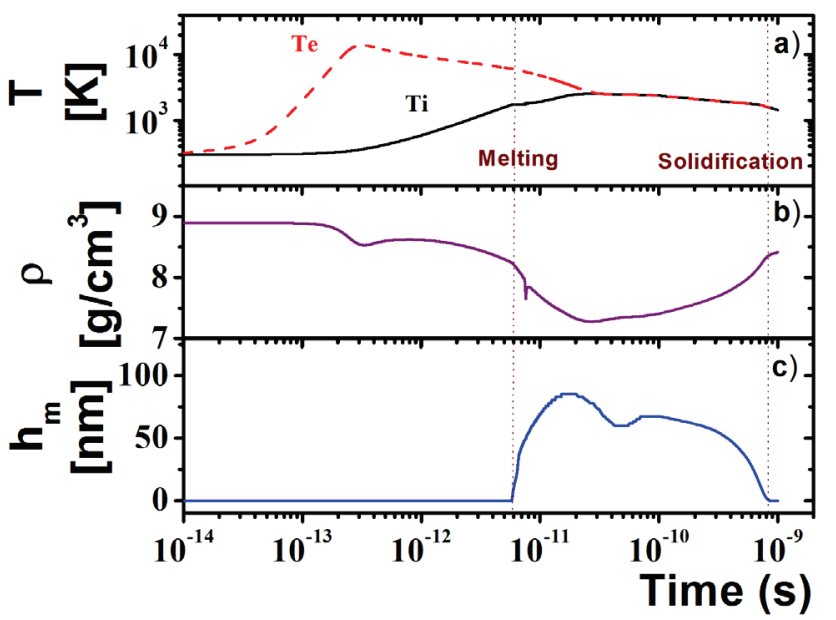

Fig. 4: Hydrodynamic calculation of the nonequilibrium temperature (a), solid-liquid phase transformation clearly visible on the density (b) and the time-varying thickness and the lifetime of the molten layer (c) of a Ni surface irradiated by a 150 fs laser pulse.

We have seen that LIPSS are readily formed upon ultrafast excitation on metals by laser pulses with a fluence sufficient to induce phase transformation. Following single- 
pulse exposure, a quasi-amorphous resolidified layer of several tens of $\mathrm{nm}$ in thickness has been experimentally observed [29-30]. These results suggest that the freezing structures morphology can depend on the thermodynamic properties of the irradiated materials. To investigate the transient thermodynamic stages of the metal surface, we have performed the simulation of the nonequilibrium heating of a nickel sample under laser irradiation using a one dimensional two temperature hydrodynamic code (Esther) [31-32]. The approach solves, according to a Lagrangian scheme, the fluid equations for the conservation of mass, momentum and energy for electronic and ionic species. The interaction between the laser field and the target is calculated by solving the Helmholtz wave equation in the inhomogeneous media. During relaxation, the material thermodynamic properties are described by the BushmanLomonosov-Fortov multiphase equation of state covering a large range of densities and temperatures between condensed states and hot plasmas [33]. This calculation enables us to monitor thermodynamic transformations of the surface, the energy profile, the onset of phase transition and hydrodynamic movement in the longitudinal direction (corresponding to laser propagation in normal incidence) to correlate them with the expected evolution of the surface topology. As a result, the evolution of thermodynamic conditions experienced by the surface is determined and depicted on Fig. 4. The figure indicates time-resolved temperature [Fig. 4(a)] and density [Fig. 4(b)] profiles of the heated surface under the action of a 150 fs pulse laser irradiation at $800 \mathrm{~nm}$ for a fluence regime allowing exceeding melting temperature at the surface but preventing an ablation process $\left(\approx 0.35 \mathrm{~J} / \mathrm{cm}^{2}\right.$ in the simulation). As a fact, LIPSS are supposed to appear only when a sufficient energy is deposited at the surface to induce liquid phase transformation and material flow, whereas for higher fluence vaporization and ablation of the surface will loosen the modulated energy deposition then inhibiting LIPSS formation. The simulations show also the kinetics of melting and solidification under ultrafast excitation. A time of a few picoseconds is required to reach the melting temperature, with the rise slope being related to the electronic temperature dependence of the electron-phonon coupling. Heat conduction to the bulk of the sample leads to cooling below the melting temperature, causing resolidification on several hundreds of picoseconds timescale at the surface. The averaged velocity of solidification can be estimated at around $80 \mathrm{~m} . \mathrm{s}^{-1}$, close to the one observed in experiments performed on $\mathrm{Ag}$ thin films heated by a fs excitation [34]. Depth of melting evolves as a function of time and reaches a maximal value of $85 \mathrm{~nm}$ at $17 \mathrm{ps}$. This time corresponds to the characteristic value for acoustic propagation at the sound velocity. Therefore, the depth of the molten zone decreases after shock relaxation but electronic thermal conduction continues to favorize the increase of the thickness of the melted layer in the following tens of picoseconds, up to around one hundred of picoseconds. We note that the size of the liquid layer is in agreement with the estimated electron diffusion depth in the solid $\mathrm{Ni}$ at the melting threshold since electronic thermal diffusion defines the heated depth in a nonequilibrium system [20]. The time scales of melting ( $\tau_{m} \approx 6 \mathrm{ps}$ ) and resolidification $\left(\tau_{s} \approx 800\right.$ ps) as well as the melting depth $\left(h_{m} \approx 80 \mathrm{~nm}\right)$ estimated in this simulation, are consistent with other calculations [35] and with most experimental observations [35-39].

\section{Modifying feedback process}

The above calculation corresponds to single pulse conditions. However in multipulse exposure conditions, the morphology of the final irradiation differs strongly from the observed single-shot one. A feedback is therefore invoked. The feedback that causes coherent structures to grow can originate from different mechanisms. LIPSS growth requires a positive feedback and we discuss in this last section possible feedback processes related to surface tension effects and plasmonic resonance alterations which can lead to non-trivial structure development.

1D-hydrodynamic calculation restricts to study in-depth effects whereas due to the large surface-to-volume ratio at nanoscale, the surface tension is supposed to play an important role in lateral organization [40]. A possible scenario of surface motion in a hydrodynamic process is that strong gradient of surface tension $\sigma$ can cause a thermocapillary force (also referred as Marangoni effect or BénardMarangoni convection) since $\sigma$ decreases as the equilibrium temperature (referred as $T$ ) increases [41]. A gradient of surface tension occurs being driven by a temperature gradient $\nabla T$ which will force the liquid to flow away from regions of low surface tension. Therefore, thermal gradients can drive the liquid flow from hotter to colder regions leading to a modulated material transport on mesoscopic scales. The time required for one convective cycle should be smaller than the total laser dwell time in terms of melt lifetime $[40,42]$. At least several pulses $(N)$ can be required to induce a significant amplitude of the generated structures and the condition of time development can be estimated to be $\tau_{c} \leq N \tau_{s}$, where $\tau_{c}$ is the characteristic time of the Marangoni flow. Supposing that surface tension is linear in temperature as $\sigma=\sigma_{0}+(\partial \sigma / \partial T) \cdot\left(T-T_{m}\right)$, where $T_{m}$ is the melting temperature. Typical values of $\tau_{c}$ can be obtained from the evaluation of the transverse velocity of a laminar liquid displacement $v_{c}[40,42-43]$ :

$$
v_{c} \approx \frac{h_{m}}{\eta}\left(\frac{\partial \sigma}{\partial T}\right)\left(\frac{\partial T}{\partial x}\right),
$$

where $\eta$ is the dynamic viscosity. Evaluating $\tau_{c}$ as $\Lambda / 2 v_{c}$ for a LIPSS spacing of $\Lambda$ with a corresponding temperature gradient $\partial T / \partial x \approx 2 \Delta T / \Lambda$ gives:

$$
\tau_{c} \approx \frac{1}{4}\left|\frac{\partial \sigma}{\partial T}\right|^{-1} \frac{\eta \Lambda^{2}}{h_{m} \Delta T} .
$$

From previous 1D hydrodynamic calculations on fs irradiated $\mathrm{Ni}$, maximal melt depth was estimated to be $h_{m} \approx 80 \mathrm{~nm}$, for a typical value of temperature rise $\Delta T \approx 10^{3} \mathrm{~K}$ corresponding to an overheating of $\approx 30 \%$ along the SP periodicity. Substituting dynamic viscosity for melted $\mathrm{Ni}$ of $\eta=4.9 \times 10^{-3}$ Pa.s [44] and $\partial \sigma / \partial T=-3.6 \times 10^{-4} \mathrm{~N} \cdot \mathrm{m}^{-1} \cdot \mathrm{K}^{-1}$ [45] in equation (3) gives 
a characteristic timescale for Marangoni flow of $\tau_{c} \approx 18 \mathrm{~ns}$ for a typical periodicity of $\Lambda=650 \mathrm{~nm}$ after multi-pulse exposure. It is clear from this estimate that $\tau_{c} / \tau_{s} \approx 20$ and that surface tension gradients contributes to move the fluid during the melt lifetime when several tens of pulses are applied. Therefore, while irradiating the metal surface by a single pulse, the thermocapillary flow would not be expected to significantly drive fluid from hot temperature regions to colder ones produced by laser inhomogeneous deposition in a periodically modulated manner. The detailed determination of Marangoni effects on an irradiated surface is beyond the scope of this paper but the above analysis suggests that a rigorous model should include this hydrodynamic process when multi-pulse experiment is considered.

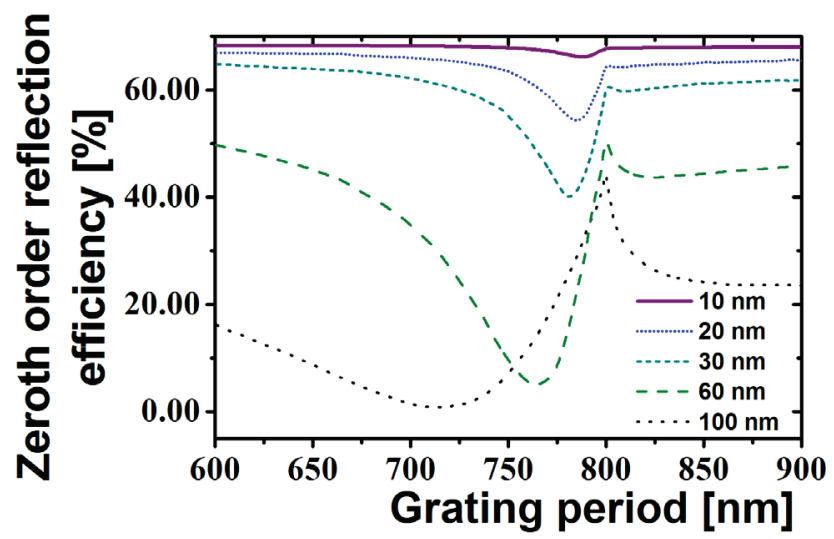

Fig. 5: Calculation of $0^{\text {th }}$-order reflection efficiency as a function of grating period for different grating depth.

It results from previous considerations that plasmonic stage does not necessarily select the dominant modulation of the final pattern since thermocapillary waves can also contribute to a nonlinear growth of specific periodicities. Nevertheless, feedback process induced by several laser shots can involve both plasmonic and capillary processes since SP are surface modes strongly localized at the surface of the metal, and therefore they are sensitive to any changes that occur in the vicinity of the air/metal interface. Indeed, the approach described in section 2 is based on a plasmonic response serving as the starting inhomogeneous absorption of the surface. This mechanism is supposed to determine both the orientation of the structures, due to a polarization dependency, and an initial periodicity which should evolve in time since LIPSS amplitude increases as the number of pulses $\mathrm{N}$ increases.

Hence, surface modification results in an increasing grating depth which, in turn, changes the surface wave dispersion. This surface modification shifts the position of the resonant grating period, where the amplitude of the shift depends on the LIPSS height. Fig. 5 illustrates the zero-th order reflection efficiency evolution from a grating with varying depth modulation from $10 \mathrm{~nm}$ to $100 \mathrm{~nm}$. The calculation has been performed for a TM wave at $800 \mathrm{~nm}$ incident on a rectangular surface grating of nickel using RCWA [25]. As it has been already shown [46], the SP resonance shifts towards a smaller periodicity for deeper gratings and a broadening of the resonance is observed. At this point, we note that a grating depth of about $75 \mathrm{~nm}$ is required to reproduce the resonance observed in our experiment at $\approx 750$ $\mathrm{nm}$ and that the shift discussed in section 2 cannot be attributed to an undesired perturbative effect. For structures deeper than $30 \mathrm{~nm}$, a large laser absorption is expected, accelerating the structuring process and promoting positive cumulative effects for a specific wavelength. Coherent structures are then supposed to develop with a dominant period related to the most enhancing or the most rapidly growing period given by the optimal multi-pulse electromagnetic and hydrodynamic feedback.

\section{Conclusion}

In conclusion we have indicated some fundamental aspects deriving from the plasmonic coupling, the electronphonon nonequilibrium stage, the subsequent sequence of phase transformation and finally the surface dynamics via surface tension gradients. Grating-coupled Surface Plasmon Polaritons on metallic surface has been exploited to investigate the correlation between LIPSS formation under ultrashort laser exposure and SP generation conditions. The main finding of this experiment was an experimental demonstration of the resonant coupling of the fs-laser pulse to the grating structure, based on SP excitation, and its impact on the early stage of LIPSS formation. The understanding of the formation dynamics of regular selforganized nanoscale structures under laser exposure of materials still requires rigorous concepts related to modulated energy deposition, laser-induced non-equilibrium phenomena and the related relaxation paths involving mass transport. Nevertheless our estimation of the Marangoni characteristic time reveals that in the particular case of strongly absorbing materials which can undergo capillary action, hydrodynamic modeling approaches should be involved to explain the observed patterns. Extended understanding effort has to be made to check if optically-induced modulation can be preserved when the system undergoes phase transitions and how feedback mechanisms can cause the growth of grating periodicities by coupling to the diffractive laser light, involving plasmonic/hydrodynamic effects.

\section{Acknowledgments}

The authors are grateful to E. Audouard for useful discussions and to S. Tonchev, N. Faure and S. Reynaud for their technical assistance. The authors are also grateful to the Program "Investissements d'Avenir"- launched by the French Government and operated by the National Research Agency (ANR) - for financial support to the LabEx MANUTECH-SISE of Université de Lyon.

\section{References}

[1] M. Birnbaum: J. Appl. Phys., 36, (1965) 3688.

[2] D. C. Emmony, R. P. Howson and L. J. Willis: Appl. Phys. Lett., 23, (1973) 598.

[3] J. Bonse, S. Baudach, J. Krüger, W. Kautek and M. Lenzner: Appl. Phys. A: Mater. Sci. Process, 74, (2002) 19. 
[4] F. Costache, S. Kouteva-Arguirova and J. Reif: Appl. Phys. A: Mater. Sci. Process., 79, (2004) 1429.

[5] J. Wang and C. Guo: Appl. Phys. Lett., 87, (2005) 251914.

[6] K. Okamuro, M. Hashida, Y. Miyasaka, Y. Ikuta, S. Tokita and S. Sakabe: Phys. Rev.B, 82, (2010) 165417.

[7] B. Dusser, Z. Sagan, H. Soder, N. Faure, J.P. Colombier, M. Jourlin and E. Audouard: Opt. Express, 18 (3) (2010) 2913.

[8] J. Bonse and J. Krüger: J. Appl. Phys., 108, (2010) 034903.

[9] A. Borowiec and H. K. Haugen: Appl. Phys. Lett., 82, (2003) 4462.

[10] R. Wagner and J. Gottmann: J. Phys.: Conf. Ser., 59, (2007) 33.

[11] V. S. Makin, R. S. Makin, A. Y. Vorobyev and C. Guo: Tech. Phys. Lett., 34, (2008) 387.

[12] R. Le Harzic, D. Dorr, D. Sauer, F. Stracke and H. Zimmermann: Appl. Phys. Lett., 98, (2011) 211905.

[13] D. J. E. Sipe, J. F. Young, J. S. Preston and H. M. van Driel: Phys. Rev. B, 27, (1983) 1141.

[14] S. A. Akhmanov, V. I. Emel'yanov, N. I. Koroteev and V. N. Seminogov: Usp. Fiz. Nauk. 147, (1985) 675 [Sov. Phys. Usp. 28, (1985) 1084].

[15]A. E. Siegman and P. M. Fauchet: IEEE J. Quantum Electron. 22, (1986) 1384.

[16] S. Sakabe, M. Hashida, S. Tokita, S. Namba and K. Okamuro: Phys. Rev. B 79, (2009) 033409.

[17] J. Reif, F. Costache, O. Varlamova, G. Jia, and M. Ratzke: Phys. Status Solidi C 6, (2009) 681.

[18]F. Garrelie, J.P. Colombier, F. Pigeon, S. Tonchev, N. Faure, M. Bounhalli, S. Reynaud and O. Parriaux: Opt. Express 19, (2011) 19150.

[19] J. Wang and C. Guo: J. Appl. Phys. 100, (2006) 023511 .

[20] J.P. Colombier, F. Garrelie, N. Faure, S. Reynaud, M. Bounhalli, E. Audouard, R. Stoian and F. Pigeon: J. Appl. Phys. 111, (2012) 024902.

[21]F. Keilmann and Y. H. Bai: Appl. Phys. A: Mater. Sci. Process. 29, (1982) 9.

[22]E. V. Golosov, A. A. Ionin, Yu. R. Kolobov, et al.: J. Exp. Theor. Phys. 113, (2011) 14.

[23]H. Raether, "Surface plasmons on smooth and rough surfaces and on gratings," Springer Tracts in Modern Physics, 111 Springer, Berlin, (1988).

[24] Handbook of optical constants of solids: ED Palik Academic, New York, (1985).

[25] N. Lyndin, O. Parriaux and A.V. Tishchenko: J. Opt. Soc. Am. A, 24, (2007) 3781.
[26] Radially Averaged Autocorrelation Function has been processed with ImageJ, a public domain java-based image processing software. http://imagejdocutudor.lu/doku.php?id=macro:radially averaged_autocorrelation

[27]Z. Lin, L. V. Zhigilei and V. Celli: Phys. Rev. B, 77, (2008) 075133.

[28] P.B. Corkum, F. Brunel, N.K. Sherman, and T. Srinivasan-Rao: Phys. Rev. Lett., 61, (1988) 2886.

[29] M. Couillard, A. Borowiec, H.K. Haugen, J.S. Preston, G.A. Botton and E.M. Griswold: J. Appl. Phys., 101 (2007) 033519.

[30] M. Schade, O. Varlamova, J. Reif, H. Blumtritt, W. Erfurthet and H. S. Leipner: Anal. Bioanal. Chem., 396 (2010), 1905.

[31] J.P. Colombier, P. Combis, F. Bonneau, R. Le Harzic and E. Audouard: Phys. Rev. B, 71, (2005) 165405.

[32] J.P. Colombier, P. Combis, E. Audouard, R. Stoian: New J. Phys. 71, (2012) 165405.

[33] A. V. Bushman, I. V. Lomonosov and V. E. Fortov: Sov. Tech. Rev. B 5, (1993) 1-44.

[34] W. L. Chan, R. S. Averback, D. G. Cahill and Y. Ashkenazy: Phys. Rev. Lett., 102, (2009) 095701.

[35] L. V. Zhigilei, Z. Lin and D. S. Ivanov: J. Phys. Chem. C, 113, (2009) 11892.

[36] S. Preuss, A. Demchuk and M. Stuke: Appl. Phys. A: Mater. Sci.Process., 61, (1995) 33.

[37] B. N. Chichkov, C. Momma, S. Nolte, F. von Alvensleben, A. Tünnermann: Appl. Phys. A: Mater. Sci. Process., 63, (1996) 109.

[38] J. Jandeleit, G. Urbasch, H. D. Hoffmann, H.-G. Treusch, E. W. Kreutz: Appl. Phys. A: Mater. Sci. Process., 63, (1996) 117.

[39] S. Nolte, C. Momma, H. Jacobs, A. Tünnermann, B. N. Chichkov, B. Wellegehausen and H. Welling: J. Opt. Soc. Am. B, 14, (1997) 2716.

[40] A. Pedraza, Y. Guan, J. Fowlkes and D. Smith: J. Vac. Sci. Technol. B, 22, (2004) 2823.

[41] S. H. Davis: Ann. Rev. Fluid Mech., 19, (1987) 403.

[42]D. Bäuerle, Laser Processing and Chemistry, 3rd ed. (Springer, Berlin, 2000).

[43] A. Ben-Yakar, A. Harkin, J. Ashmore, R. Byer and H. Stone: J. Appl. Phys. D, 40, (2007) 1447.

[44]F. J. Cherne, M. I. Baskes and P. A. Deymier: Phys. Rev. B, 65, (2001) 024209.

[45]I. Egry, G. Lohoefer and G. Jacobs: Phys. Rev. Lett., 75, (1995) 4043.

[46] M. Huang, F. Zhao, Y. Cheng, N. Xu and Z. Xu: ACS Nano 3(12), (2009) 4062.

(Received: June 29, 2012, Accepted: October 26, 2012) 